\title{
A New 3D Simulation Method for the Construction of Optical Phase Contrast Images of Gold Nanoparticle Clusters in Biological Cells
}

\author{
Stoyan Tanev, ${ }^{1}$ James Pond, ${ }^{2}$ Paul Paddon, ${ }^{2}$ and Valery V. Tuchin ${ }^{3,4}$ \\ ${ }^{1}$ Department of Systems and Computer Engineering, Carleton University, 1125 Colonel By Drive, Ottawa, ON, Canada K1S $5 B 6$ \\ ${ }^{2}$ Lumerical Solutions, Inc., Vancouver, BC, Canada V6B $2 Y 5$ \\ ${ }^{3}$ Research-Educational Institute of Optics and Biophotonics, Saratov State University, 410012 Saratov, Russia \\ ${ }^{4}$ Institute of Precise Mechanics and Control of RAS, 410028 Saratov, Russia \\ Correspondence should be addressed to Stoyan Tanev, tanev@sce.carleton.ca
}

Received 5 June 2008; Accepted 30 October 2008

Recommended by Brian Wilson

\begin{abstract}
A new 3D simulation method based on the finite-difference time domain (FDTD) approach in combination with Fourier optic techniques is applied to the modeling of optical phase contrast microscope (OPCM) imaging of gold nanoparticles (NPs) in singe biological cells. We consider a realistic size 3D cell model at optical immersion conditions, that is, when the refractive index values of the cytoplasm and of the extracellular medium are equal. For the first time, an FDTD-based OPCM model is applied to visualize the presence of a cluster of gold NPs in the cytoplasm at both resonant and nonresonant conditions. The results demonstrate the capability to model OPCM image enhancement by optically controlling the resonant properties of the NPs. Our research study extends the applicability of the FDTD modeling approach into a new biomedical optics research area.
\end{abstract}

Copyright (C) 2008 Stoyan Tanev et al. This is an open access article distributed under the Creative Commons Attribution License, which permits unrestricted use, distribution, and reproduction in any medium, provided the original work is properly cited.

\section{INTRODUCTION}

One of the growing areas in biomedical optics and photonics $[1,2]$ is the application of optical software simulation and modeling tools to provide deeper understanding of newly developed optical diagnostics and imaging techniques. There are a number of numerical modeling approaches that can be used for the modeling of the light scattering from biological cells [3] including the finite-difference time domain (FDTD) method [4-9]. Recently, we demonstrated the applicability of the 2D FDTD approach to numerically study the changes in the forward scattered light phase and intensity distributions due to different cell membrane thicknesses and different configurations of gold NPs in the cytoplasm of small size cells using the optical immersion technique (OIT) $[7,8]$. The OIT is associated with the so-called "optical clearing" (OC) effect consisting in the increased light transmission through cells or tissues due to the matching of the refractive indices of some of their morphological components to that of the extracellular or interstitial media [10-12]. In this article, we present the first results of a research study applying a 3D FDTD-based optical phase contrast microscope (OPCM) imaging approach to the modeling of a cluster of gold nanoparticles (NPs) in realistic size single cells. We demonstrate a new simple way of using the FDTD approach for the construction of OPCM images by moving its modeling power from the calculation of phase distribution curves [7] to realistic cell images. We validate our approach by modeling a gold nanoparticle cluster in the cell cytoplasm to provide a valuable design methodology for further studies in optical nano-bioimaging and nanotherapeutics.

\section{THE FDTD APPROACH}

The FDTD technique is a numerical solution of Maxwell's equations [13]. In most light scattering simulation experiments, one usually uses the so-called total field/scattered field (TFSF) formulation $[8,13]$ to excite the magnetic and electric fields and simulate a linearly polarized plane wave propagating in a finite region containing the simulation equivalent of the object under investigation [13]. 


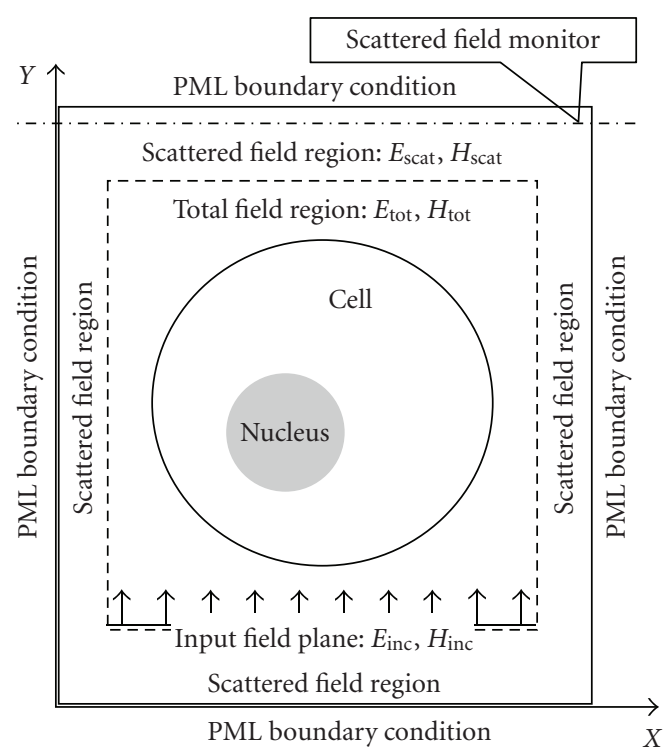

(a)

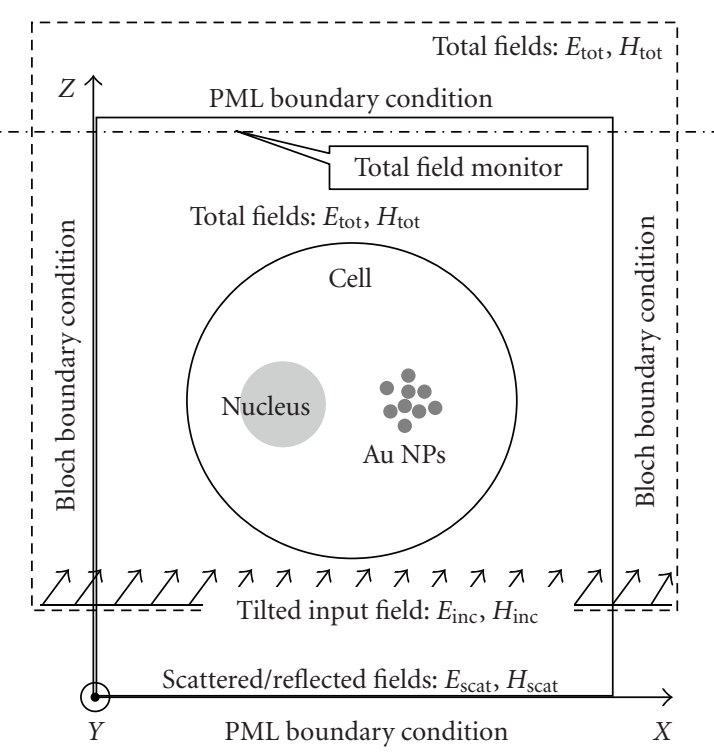

(b)

FIGURE 1: Schematic representation of the FDTD computational domain: (a) total field/scattered field 2D formulation; (b) total field/reflected field 3D formulation.

A schematic representation of the 2D FDTD computational domain is shown in Figure 1(a). TFSF sources are used to separate the FDTD computation domain into two distinct regions. The first one is internal to the computational domain and contains the total near fields $E_{\mathrm{tot}}=E_{\mathrm{inc}}+E_{\mathrm{scat}}$ and $H_{\text {tot }}=H_{\text {inc }}+H_{\text {scat }}$, that is, the sum of the incident field and the scattered near field. The second one occupies the rest of the computational domain and contains only the scattered near fields $E_{\text {scat }}=E_{\text {tot }}-E_{\text {inc }}$ and $H_{\text {scat }}=H_{\text {tot }}-$ $H_{\text {inc }}$. The final FDTD simulation results require additional postprocessing computational procedures to transform the real values of the calculated near fields into their complex far field counterparts. The far fields include the transverse distribution of the forward scattered light from the cell which is of high relevance for the modeling of optical phase contrast imaging. At the edges, the entire FDTD computational domain is truncated by the so-called perfectly matched layer (PML) boundary conditions [13].

The 3D case of the TFSF formulation described above follows a similar logic. The $3 \mathrm{D}$ simulation results provided here are based on a modified 3D TFSF formulation that could be more appropriately called total field/reflected field (TFRF). The 3D "TFRF" formulation uses a TFSF region which contains the biological cell and extends beyond the limits of the simulation domain (Figure 1(b)). The extension of the transverse dimension of the input field beyond the limits of the computational domain through the PML boundaries would lead to distortions of its ideal plane wave shape and eventually distort the simulation results. To avoid these distortions, one must use Bloch periodic boundary conditions (Figure 1(b)) in the lateral $x$ - and $y$-directions which are perpendicular to the direction of propagation$z$ [13]. Bloch boundary conditions are periodic boundary conditions which take into account the phase effects due to the tilting of the input plane waves incoming at periodic structures, that is, what we are actually modeling is a periodic row of biological cells. The near scattered fields, however, are calculated in the transverse planes located in the close proximity to the cell where the coupling effect due to waves scattered from adjacent cells is negligible. This effect can be further minimized or completely removed by controlling the lateral dimension of computational domain by using a large enough period of the periodic cell structure. The larger is this period, the smaller is the coupling effect. In the 3D TFRF formulation, the location in the computational domain corresponding to the forward scattered light is positioned within the total field region (Figure 1(b)). The OPCM simulation model requires the explicit availability of the forward scattered transverse distribution of the fields. The phase of the scattered field accumulated by a plane wave propagating through a biological cell will be used in the FDTD model of the OPCM that will be described in Section 3.

\section{OPTICAL PHASE CONTRAST MICROSCOPE MODEL}

\subsection{FDTD OPCM principle}

Phase contrast microscopy is utilized to produce highcontrast images of transparent specimens such as microorganisms, thin tissue slices, living cells, and subcellular components such as nuclei and organelles. It translates smallphase variations into corresponding changes in amplitude visualized as differences in image contrast. A standard phase contrast microscope design is shown in Figure 2(a), where an image with a strong contrast ratio is created by coherently interfering a reference $(R)$ with a diffracted beam $(D)$ from the specimen (http://www.microscopyu.com/articles/phasecontrast/phasemicroscopy.html). Relative to the reference 


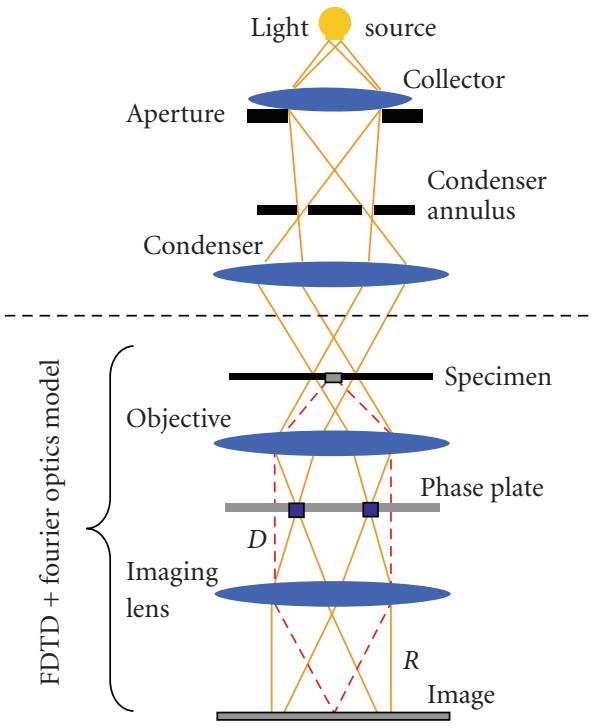

(a)

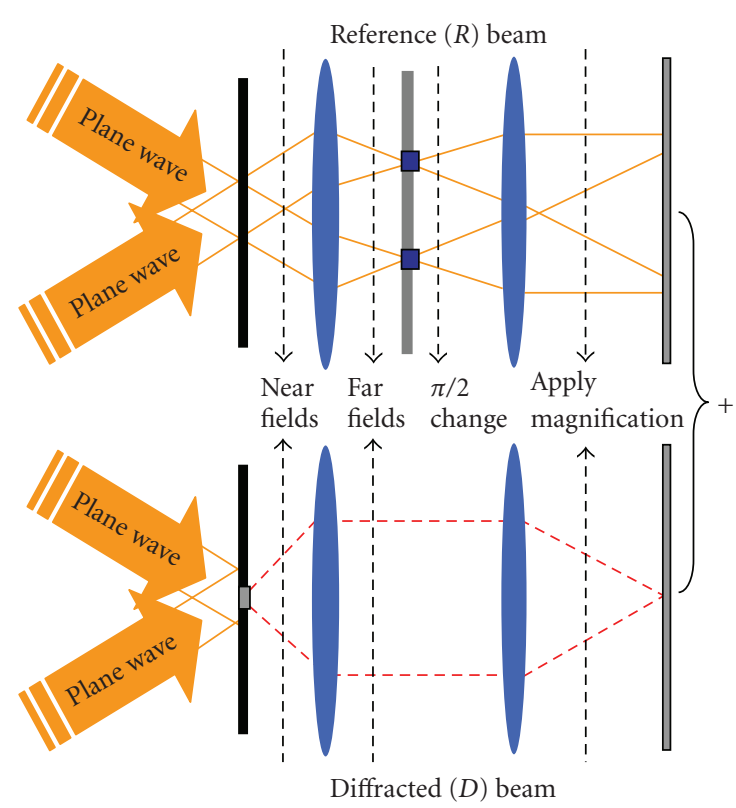

(b)

FIGURE 2: (a) Schematic representation of an OPCM. (b) 2D visual representation of the FDTD OPCM model using incoherent illumination by two planes waves at a polar angle of 30 degrees. For each of the two plane waves, the propagation of light is modeled as a combination of two parallel wave phenomena: (i) propagation of the reference $(R)$ beam without the cell, and (ii) propagation of the diffracted $(D)$ beam due to the cell.

beam, the diffracted beam has lower amplitude and is retarded in phase by approximately $\pi / 2$ through interaction with the specimen.

The main concept underlying the design of the phase contrast microscope is the spatial separation of the $R$ beam from $D$ wave front emerging from the specimen. In addition, the amplitude of the $R$ beam light must be reduced and the phase should be advanced or retarded by another $\pm \pi / 2$ in order to maximize the differences in the intensity between the specimen and the background in the image plane. The mechanism for generating relative phase retardation has two steps: (i) the $D$ beam is being retarded in phase by a quarter wavelength (i.e., $\pi / 2$ ) at the specimen, and (ii) the $R$ beam is advanced (or retarded) in phase by a phase plate positioned in or very near the objective rear focal plane (Figure 2(a)). This two-step process is enabled by a specially designed annular diaphragm - the condenser annulus - which is placed in the condenser front focal plane, is matched in diameter, and optically conjugates to the phase plate residing in the objective rear focal plane. The resulting image, where the total phase difference is translated by interference at the image plane into an amplitude variation, can have a high contrast ratio, particularly if both beams have the same amplitude.

\subsection{Excitation and simulation of the FDTD near fields}

Figure 2(a) illustrates the part of the microscope that will become the subject of FDTD modeling combined with Fourier optics. Figure 2(b) provides a visual representation illustrating the major steps in the FDTD OPCM model. The phase contrast microscope uses incoherent annular illumination that could be approximately modeled by adding up the results of eight different simulations using ideal input plane waves incident at a given polar angle (30 degrees), an azimuthal angle $(0,90,180$, or 270 degrees), and a specific light polarization (parallel or perpendicular to the plane of the graph). Every single FDTD simulation provides the near field components in a transverse monitoring plane located right behind the cell (Figure 1(b)).

\subsection{Far-field transformation}

The far field transformations (Figure 3) use the FDTD calculated near fields right behind the cell and return the three complex components of the electromagnetic fields at 1 meter distance (i.e., long enough) from the location of the near fields, that is, in the far field $[13,14]$ : $E_{r}\left(u_{x}, u_{y}\right), E_{\theta}\left(u_{x}, u_{y}\right)$, and $E_{\Phi}\left(u_{x}, u_{y}\right)$, where $(r, \theta, \Phi)$ refer to a spherical coordinate system and the variables $u_{x}$ and $u_{y}$ are the $x$ and $y$ components of the unit direction vector $\mathbf{u}$. The unit direction vector is related to the angular variables by $u_{x}=\sin (\theta) \cos (\Phi), u_{y}=\sin (\theta) \sin (\Phi), u_{z}=\cos (\theta)$, with $u_{x}^{2}+u_{y}^{2}+u_{z}^{2}=1$. The in-plane wave vectors for each plane wave are given by $k_{x}=k u_{x}$ and $k_{y}=k u_{y}$, where $k=2 \pi / \lambda$. To scale the far field to a different distance, we can recognize that in $3 \mathrm{D}$, the electric field intensity decreases like $1 / R^{2}$ : $|E(R)|^{2}=\left|E_{0}\right|^{2} / R^{2}$, where $E_{0}$ is the field at $1 \mathrm{~m}$ distance resulting from the far field transformation and $R$ is measured 


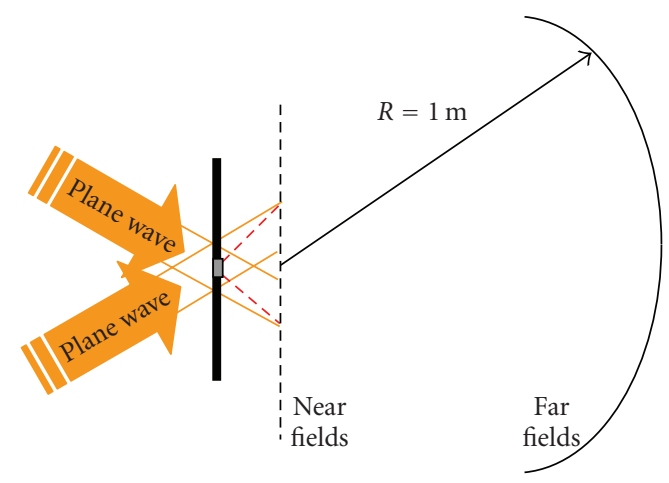

FIGURE 3: Visual representation of the far field transformation procedure within the context of the optical phase contrast microscope schematics presented in Figures 2.

in meters. The specific details of the far field transformation can be found elsewhere [13].

It is important to note that the near-to-far field projection must take into account the Bloch periodic boundary conditions in the lateral dimension and is calculated only at angles that correspond to diffracted orders of the periodic structure as defined by the Bragg conditions. This is done by calculating the direction cosines of all the diffracted orders that meet the Bragg condition, and interpolating the previously far-field distributions onto those specific directions. The near-to-far field projection, therefore, provides the electric field amplitude and phase corresponding to each diffracted order. The zeroth order, that is, the light travelling through the scattering object without any deviation in angle, is the reference beam; and the phase contrast microscope is designed to provide a phase delay to this order.

\subsection{Optical magnification}

Now that we have the field components $\mathbf{E}\left(k_{x}, k_{y}\right)$, we can use their amplitudes and phases to determine all the properties of the polarization as well as do Fourier optics for both the scattered and reference beams. We assume an ideal optical lens system that could be characterized by a given magnification factor. This simple model could be easily extended to include the numerical equivalent of the two lenses (Figure 2) together with an additional model to take into account any aberrational effects. The magnification was implemented by modifying the angle of light propagation, that is, by multiplying the direction cosines $u_{x}$ and $u_{y}$ by the inverse value of the desired magnification factor $M$ : $U_{x}=u_{x} / M$ and $U_{y}=u_{y} / M$. In any other circumstances, the modification of the direction cosines would lead to complications because of the vectorial nature of the $E$ field. In our case, however, working in spherical coordinates $\left(E_{r}, E_{\theta}\right.$, and $\left.E_{\Phi}\right)$ leads to the advantage that the vectorial components do not change when $u_{x}$ and $u_{y}$ are modified because they are part of a local coordinate system that is tied to the values of $u_{x}$ and $u_{y}$. The magnification factor $M=10$ was applied to the far fields before the interference of the diffracted $(D)$ and reference $(R)$ beams at the image plane.

\subsection{Numerical aperture}

It was also possible to apply the effect of a numerical aperture $\mathrm{NA}=0.8$ which clips any light that has too steep angle and would not be collected by the lens system. This means that all the light with $U_{x}^{2}+U_{y}^{2}>\mathrm{NA}^{2}$ is being clipped. The effect of the aperture is defined by the inequality $U_{x}^{2}+U_{y}^{2}>\mathrm{NA}^{2}$ applied to the corrected aperture angles $\theta^{\prime}$ and $\Phi^{\prime}$ :

$$
\begin{aligned}
\sin \left(\Phi^{\prime}\right) & =\frac{U_{y}}{U_{x y}}, \\
\cos \left(\Phi^{\prime}\right) & =\frac{U_{x}}{U_{x y}}, \\
\cos \left(\theta^{\prime}\right) & =U_{z}, \\
\sin \left(\theta^{\prime}\right) & =U_{x y},
\end{aligned}
$$

where $U_{x y}=\operatorname{sqrt}\left(U_{x}^{2}+U_{y}^{2}\right), U_{z}=\operatorname{sqrt}\left(1-U_{x y}^{2}\right)$ and the "sqrt" labels a square root mathematical operation. The magnified field components will then have the following form.

Diffracted $(D)$ beam:

$$
\begin{aligned}
& E_{x-D}\left(k_{x}, k_{y}\right)=-E_{\Phi} \sin \left(\Phi^{\prime}\right)+E_{\theta} \cos \left(\Phi^{\prime}\right) \cos \left(\theta^{\prime}\right), \\
& E_{y-D}\left(k_{x}, k_{y}\right)=E_{\Phi} \cos \left(\Phi^{\prime}\right)+E_{\theta} \sin \left(\Phi^{\prime}\right) \cos \left(\theta^{\prime}\right), \\
& E_{z-D}\left(k_{x}, k_{y}\right)=-E_{\theta} \sin \left(\theta^{\prime}\right) .
\end{aligned}
$$

\section{Reference $(R)$ beam:}

$$
\begin{aligned}
& E_{x-R}\left(k_{x}, k_{y}\right)=-E_{\Phi-R} \sin \left(\Phi^{\prime}\right)+E_{\theta-R} \cos \left(\Phi^{\prime}\right) \cos \left(\theta^{\prime}\right), \\
& E_{y-R}\left(k_{x}, k_{y}\right)=E_{\Phi-R} \cos \left(\Phi^{\prime}\right)+E_{\theta-R} \sin \left(\Phi^{\prime}\right) \cos \left(\theta^{\prime}\right), \\
& E_{z-R}\left(k_{x}, k_{y}\right)=-E_{\theta-R} \sin \left(\theta^{\prime}\right),
\end{aligned}
$$

where $E_{\theta-R}$ are $E_{\Phi-R}$ are the far field components of the reference beam.

\subsection{OPCM image construction}

The fields given above are then used to calculate back the Fourier inverse transform of the far field transformed fields leading to the distribution of the scattered and the references beams in the image plane.

\section{Diffracted $(D)$ beam:}

$$
\begin{aligned}
& E_{x-D}=\operatorname{sum}\left(E_{x-D}\left(k_{x}, k_{y}\right) \exp \left(i k_{x} x+i k_{y} y+i k_{z} z\right)\right), \\
& E_{y-D}=\operatorname{sum}\left(E_{y-D}\left(k_{x}, k_{y}\right) \exp \left(i k_{x} x+i k_{y} y+i k_{z} z\right)\right), \\
& E_{z-D}=\operatorname{sum}\left(E_{z-D}\left(k_{x}, k_{y}\right) \exp \left(i k_{x} x+i k_{y} y+i k_{z} z\right)\right) .
\end{aligned}
$$

\section{Reference $(R)$ beam:}

$$
\begin{aligned}
& E_{x-R}=\operatorname{sum}\left(E_{x-R}\left(k_{x}, k_{y}\right) \exp \left(i k_{x} x+i k_{y} y+i k_{z} z\right)\right), \\
& E_{y-R}=\operatorname{sum}\left(E_{y-R}\left(k_{x}, k_{y}\right) \exp \left(i k_{x} x+i k_{y} y+i k_{z} z\right)\right), \\
& E_{z-R}=\operatorname{sum}\left(E_{z-R}\left(k_{x}, k_{y}\right) \exp \left(i k_{x} x+i k_{y} y+i k_{z} z\right)\right),
\end{aligned}
$$

where the summation is over all angles. 
The OPCM images at the image plane are calculated by adding up the scattered and the reference beam at any desired phase offset $\Psi$ :

$$
\begin{aligned}
I= & \operatorname{abs}\left(E_{x-D}+a E_{x-R} \exp (i \Psi)\right)^{2} \\
& +\operatorname{abs}\left(E_{y-D}+a E_{y-R} \exp (i \Psi)\right)^{2} \\
& +\operatorname{abs}\left(E_{z-D}+a E_{z-R} \exp (i \Psi)\right)^{2} .
\end{aligned}
$$

The coefficient $a$ and the phase $\Psi$ correspond to the ability of the OPCM to adjust the relative amplitudes and the phase difference between the two beams. In all simulation results presented in this article, $a=1$ and $\Psi$ is used as parameter.

\section{CELL STRUCTURE, GOLD NANOPARTICLE MODEL AND SIMULATION RESULTS}

\subsection{Cell structure}

The cell is modeled as a dielectric sphere with a realistic radius $R_{c}=5 \mu \mathrm{m}$. It is critically important to pay special attention to the membrane model since assigning the membrane the precisely desired thickness value leads to inadequate modeling of optical immersion experiments. This is due to the fact that the FDTD technique averages out the refractive index values at the interface of two different materials effectively reducing the membrane thickness value and eventually, due to the staircase approximation error, destroying the continuity of the membrane. This is the reason for us to select a membrane thickness $d=20 \mathrm{~nm}$ which corresponds to effective thickness of approximately $10 \mathrm{~nm}$. The cell nucleus is also spherical with a radius $R_{n}=1.5 \mu \mathrm{m}$ centered at a position which is $2.0 \mu \mathrm{m}$ shifted from the cell center in a direction perpendicular to the direction of light propagation. The refractive index of the cytoplasm is $n_{\text {cyto }}=$ 1.36 , of the nucleus $n_{\text {nuc }}=1.4$, of the membrane $n_{\text {mem }}=$ 1.47 , and of the extracellular material $n_{\text {ext }}=1.33$ (no refractive index matching) or 1.36 (refractive index matching).

\subsection{Optical properties of gold nanoparticles}

Gold NPs exhibit theability to resonantly scatter visible and near infrared light due to the excitation of surface plasmonresonances (SPRs). The scattering ability is extremely sensitive to their size, shape, andaggregation state offering a great potential for optical cellular imaging and detection labeling studies [15-19]. Our FDTD approach uses the dispersion model for gold derived from the experimental data provided by Johnson and Christy $[7,20]$ where the total, complex-valued permittivity is given as

$$
\varepsilon(\omega)=\varepsilon_{\mathrm{REAL}}+\varepsilon_{L}(\omega)+\varepsilon_{P}(\omega)
$$

Each of the three contributions to the permittivity arises from a different material model. The first term represents the contribution due to the basic, background permittivity.
The second and third terms represent Lorentz and plasma contributions:

$$
\begin{aligned}
& \varepsilon_{L}(\omega)=\frac{\varepsilon_{\text {LORENTZ }} \omega_{0}^{2}}{\omega_{0}^{2}-2 i \delta_{0} \omega-\omega^{2}}, \\
& \varepsilon_{P}(\omega)=\frac{\omega_{P}^{2}}{i \omega \nu_{C}+\omega^{2}},
\end{aligned}
$$

where all material constants are summarized in Table 1.

We have modeled two different cases-resonant and nonresonant. The ability to model both these cases together with the effect of optical clearing effect provides the opportunity to numerically study the possibility for imaging of the uptake of clusters of NPs - a scenario which, to the best of our knowledge, was not studied before. We used the FDTD technique to calculate the scattering and absorption crosssections over a 400-900 nm wavelength range for a single $50 \mathrm{~nm}$ diameter gold NP immersed in a material having the properties of the cytoplasm $(n=1.36)$ and resolution $d x=$ $d y=d z=10 \mathrm{~nm}$. The scattering cross-section is defined as

$$
\sigma_{\text {scat }}=\frac{P_{\text {scat }}(\omega)}{I_{\text {inc }}(\omega)},
$$

where $P_{\text {scat }}$ is the total scattered power and $I_{\text {inc }}$ is the intensity of the incident light in $\left(\mathrm{W} / \mathrm{m}^{2}\right)$. It was calculated by applying the total field/scattered field FDTD formulation [13] and by creating 12 field power monitoring planes around the nanoparticle in the form of a box: 6 in the total field region and 6 in the scattered field region. The total scattered power is calculated by summing up the power flowing outward through 6 scattered field power monitors located in the scattered field region.

The absorption cross-section is similarly defined as

$$
\sigma_{\mathrm{abs}}=\frac{P_{\mathrm{abs}}(\omega)}{I_{\mathrm{inc}}(\omega)},
$$

where $P_{\mathrm{abs}}$ is the total power absorbed by the particle. The power absorbed by the particle is calculated by calculating the net power flowing inward through the 6 total field power monitors located in the total field region. The extinction cross-section is the sum of the absorption and scattering cross-sections

$$
\sigma_{\mathrm{ext}}=\sigma_{\mathrm{scat}}+\sigma_{\mathrm{abs}}
$$

Figure 4 shows that the extinction cross-section has a maximum of 3.89 at around $543.0 \mathrm{~nm}$ corresponding to one of the radiation wavelengths of He-Ne lasers. In this article, we also present results for $\lambda=676.4 \mathrm{~nm}$ (a Krypton laser wavelength) which corresponds to the nonresonant case (extinction cross-section value $0.322, \sim 12$ times smaller than 3.89). The FDTD results are compared with the theoretical curve calculated by Mie theory. The main reason for the slight discrepancy between the theoretical and FDTD results for the extinction cross-section is the finite mesh size.

To consistency of the results could be improved by reducing the mesh size. In the FDTD simulations including both the cell and the nanoparticles, the numerical resolution 
TABLE 1: Optical material constants of gold [20].

\begin{tabular}{ccc}
\hline Background permittivity & Lorentz dispersion & Plasma dispersion \\
\hline$\varepsilon_{\text {LORENTZ }}=2.323$ & $\omega_{P}=1.391 \times 10^{16} \mathrm{~Hz}$ \\
$\mathcal{E}_{\text {REAL }}=7.077$ & $\omega_{0}=4.635 \times 10^{15} \mathrm{~Hz}$ & $\nu_{C}=1.411 \times 10^{7} \mathrm{~Hz}$ \\
& $\delta_{0}=9.267 \times 10^{14} \mathrm{~Hz}$ & \\
\hline
\end{tabular}

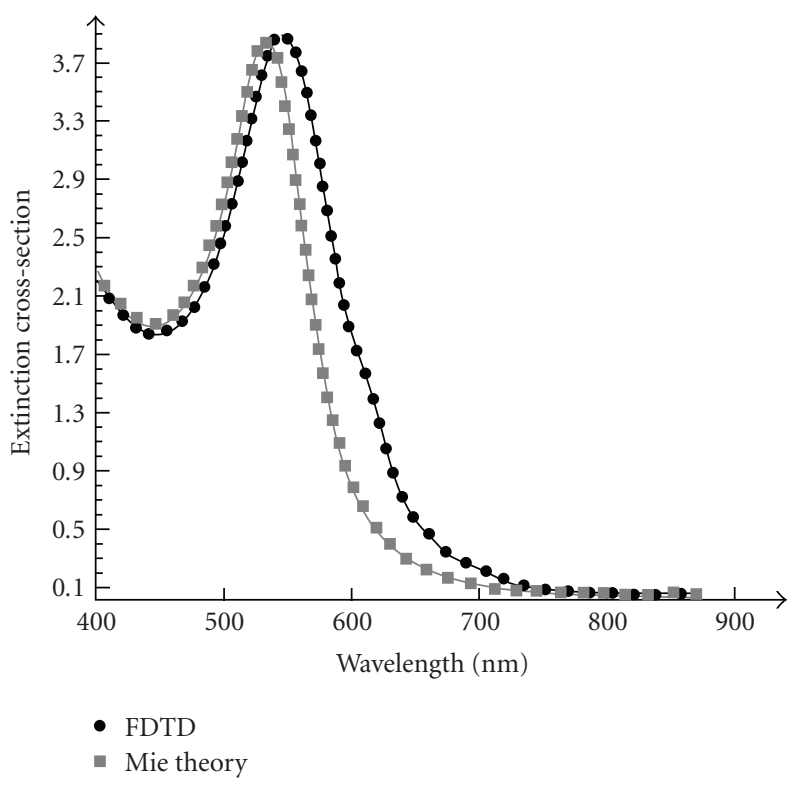

FIGURE 4: Extinction cross-section of a $50 \mathrm{~nm}$ gold nanoparticle immersed in material having the optical properties of the cytoplasm: $n=1.36$. The optical properties of gold are described by (7) and (8) and the parameters given in Table 1.
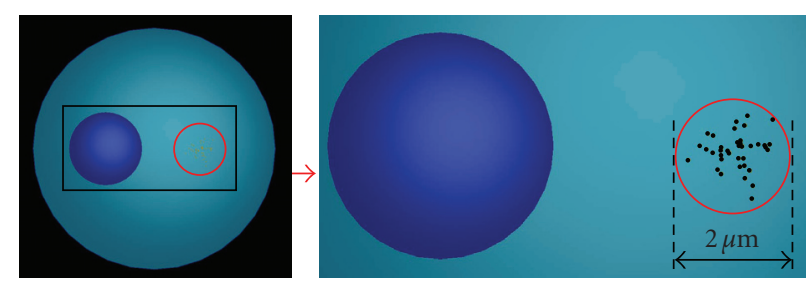

FIgURE 5: A cluster of 42 gold nanoparticles randomly distributed in a spatial sphere with a radius of $1 \mu \mathrm{m}$ at a cell location opposite to the location of the nucleus $(x=2 \mu \mathrm{m}, y=0, z=0)$. The nucleus is located at $x=-2 \mu \mathrm{m}$. The gold nanoparticle size (diameter $=50 \mathrm{~nm})$ on right-hand graph is slightly exaggerated.

of the nanoparticles was hard-coded to $d x=d y=d z=$ $10 \mathrm{~nm}$ to make sure that their numerically manifested optical resonant properties will be the same as the ones shown in Figure 4. However, Figure 5 visualizes the positioning of a cluster of 42 nanoparticles in the cytoplasm that was used to produce the FDTD simulation results presented in Section 5. It was randomly generated without controlling the possibility for spatial overlaps or contacts between the nanoparticles.
The closer visual inspection showed that there were no such overlaps and contacts.

\section{3. $O P C M$ image simulation results}

The simulation results presented here are based on the 3D FDTD OPCM model. The simulations use nonuniform meshing where the number of mesh points in space is automatically calculated to ensure a higher number of mesh points in materials with higher values of the refractive index [14]. The realistic cell dimensions (including both cell radius and membrane) require a very fine numerical resolution making the simulations computationally intensive. The locally refined resolution of the nanoparticles leads to additional requirements for the CPU time and memory $(\sim 120$ Gbs RAM) requiring high performance computing resources. The time step used during the simulation was defined by means of a factor of 0.99 in the Courant stability limit: $c \Delta t=0.99 \times\left(1 / \Delta x^{2}+1 / \Delta y^{2}+1 / \Delta z^{2}\right)^{-1 / 2}$. The cell center is located in the middle $(x=y=z=0)$ of the computational domain with dimensions $15 \mu \mathrm{m} \times 12 \mu \mathrm{m} \times$ $15 \mu \mathrm{m}$. The nucleus' center is located at $x=-2 \mu \mathrm{m}, y=$ $z=0 \mu \mathrm{m}$. The cluster of gold nanoparticles is located at $x=2 \mu \mathrm{m}, y=z=0 \mu \mathrm{m}$ (Figure 5).

The refractive index matching (RIM) between the cytoplasm and the extracellular medium leads to the optical clearing of the image and a significantly better OPCM image of the nucleus and of the membrane-the only sources of phase contrast at optical immersion conditions. Based on the fact that RIM enhances significantly the imaging of the cell components, we have used the FDTD OPCM model to create the OPCM images of the same cell at optical immersion conditions including the cluster of 42 gold NPs (Figure 5, see also Figure 1(b)) and for different phase offsets between the reference beam and the scattered beam (assuming $a=$ 1). The insertion of the nanoparticles increases significantly the CPU and memory requirements. The dimensions of the computational domain are $15 \mu \mathrm{m} \times 15 \mu \mathrm{m} \times 12 \mu \mathrm{m}$. The OPCM images in Figure 6 show that the gold NP cluster can be clearly identified at both the nonresonant $(\lambda=676.4 \mathrm{~nm})$ and resonant $(\lambda=543.0 \mathrm{~nm})$ wavelengths.

The graphs in Figure 7 compare the geometrical crosssections of the three OPCM images shown at the bottom of Figure 6 at $y=0 \mu \mathrm{m}$ showing the relevant half of the images (right) where the gold NP cluster is located. At resonance, the optical contrast of the gold NP peak is $\sim 2.24$ times larger than the one at no resonance and 24.79 times larger than the background optical contrast corresponding to the case when there are no nanoparticles. The enhanced imaging of 
RIM, no NPs, $\lambda=676.4 \mathrm{~nm}$
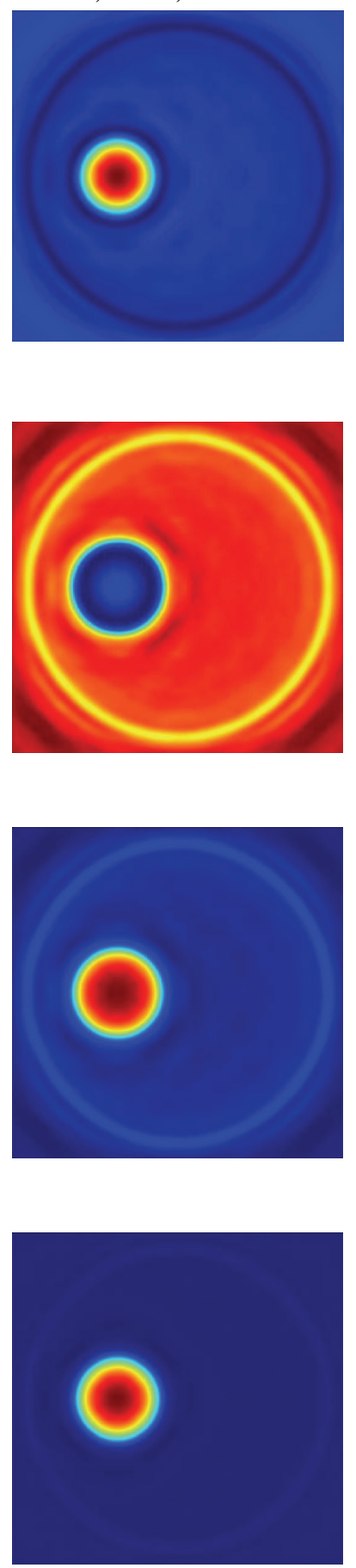

RIM, NPs, $\lambda=676.4 \mathrm{~nm}$

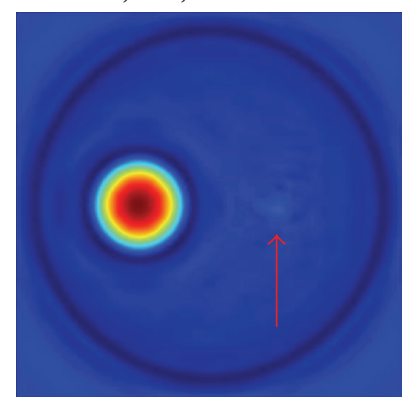

(a)

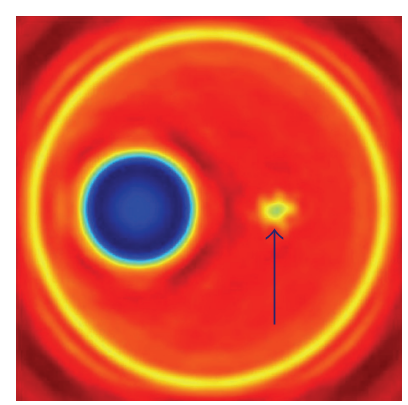

(b)

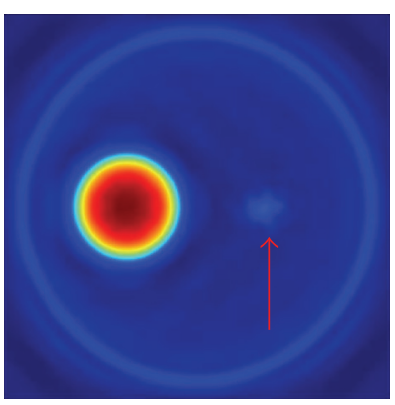

(c)

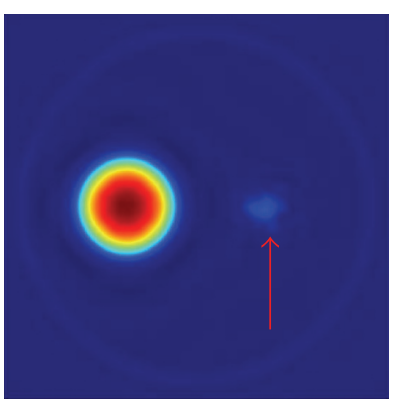

(d)
RIM, NPs, $\lambda=543 \mathrm{~nm}$
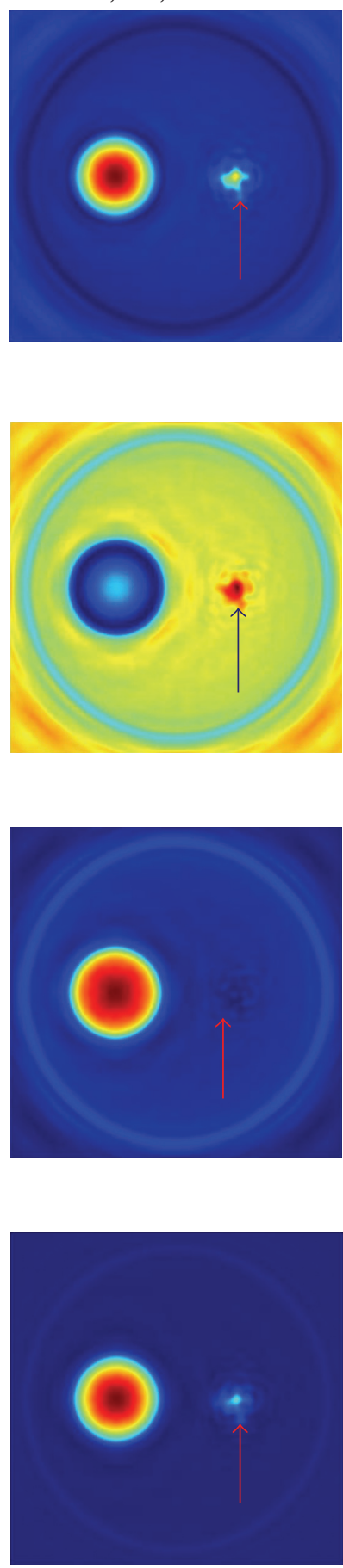

FIGURE 6: OPCM images of a biological cell for different values (a: $\left.-150^{\circ}, \mathrm{b}:-90^{\circ}, \mathrm{c}: 90^{\circ}, \mathrm{d}: 180^{\circ}\right)$ of the phase offset between the reference and diffracted beam of the OPCM at optical immersion conditions (left) including a cluster of 42 gold NPs (middle: no resonance, right: at resonance) located in a position symmetrically opposite to the nucleus. The arrows indicate the position of the gold NP cluster.

the gold NP cluster at resonant conditions is of no surprise. It, however, needs to be further studied as a function of particular phase delay $\Psi$ between the reference beam and the scattered beam. Figure 8 visualizes the optical contrast due to the gold nanoparticle cluster as a function of the phase offsets between the reference $(R)$ and the diffracted $(D)$ beams of the optical phase microscope. It shows that the enhancement of the optical contrast due to the nanoparticle resonance changes significantly from a minimum of $0.0\left(\Psi=0^{\circ}\right)$ to a maximum of $3.60\left(\Psi=-150^{\circ}\right)$. 


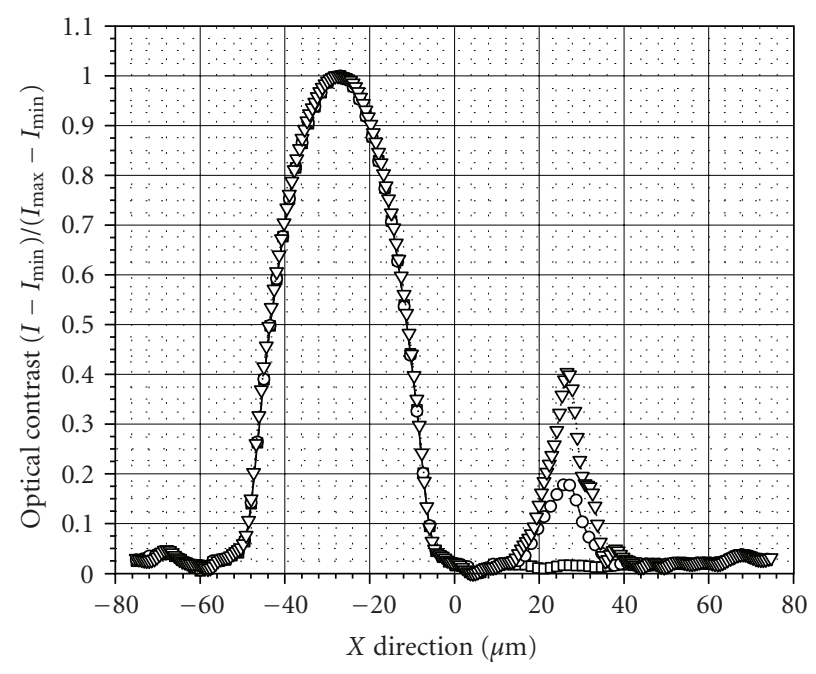

(a)

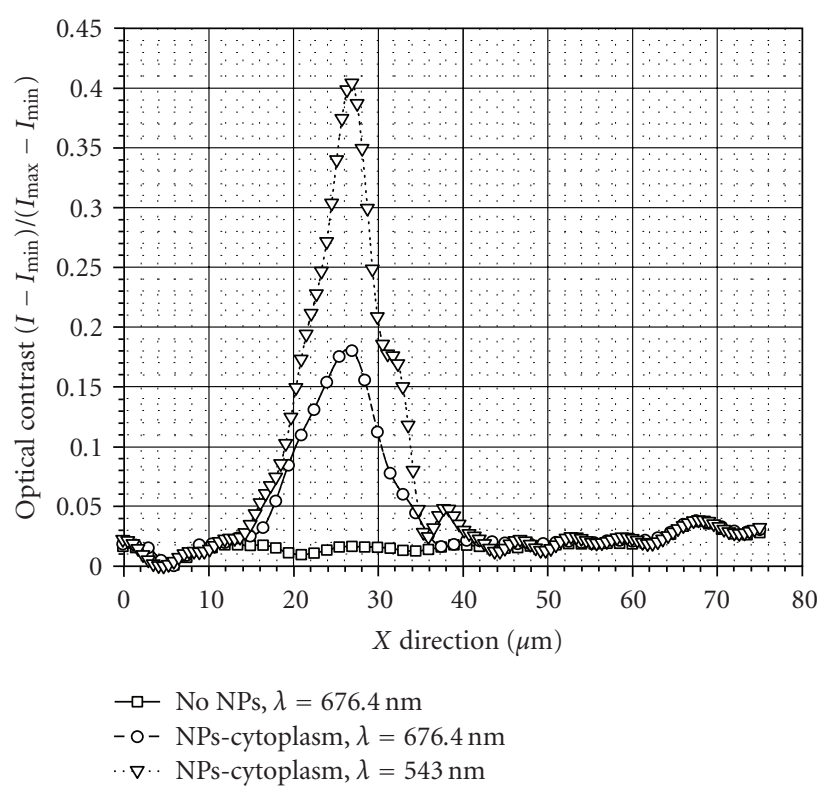

(b)

FIGURE 7: Comparison of the geometrical cross sections at $y=0 \mu \mathrm{m}$ of the three OPCM images corresponding to $180^{\circ}$ phase delay between the reference and the diffracted beam (bottom row in Figure 6) in terms of optical contrast. The right-hand side graph illustrates the optical contrast enhancement due to the effect of the gold NP resonance at $\lambda=543 \mathrm{~nm}$.

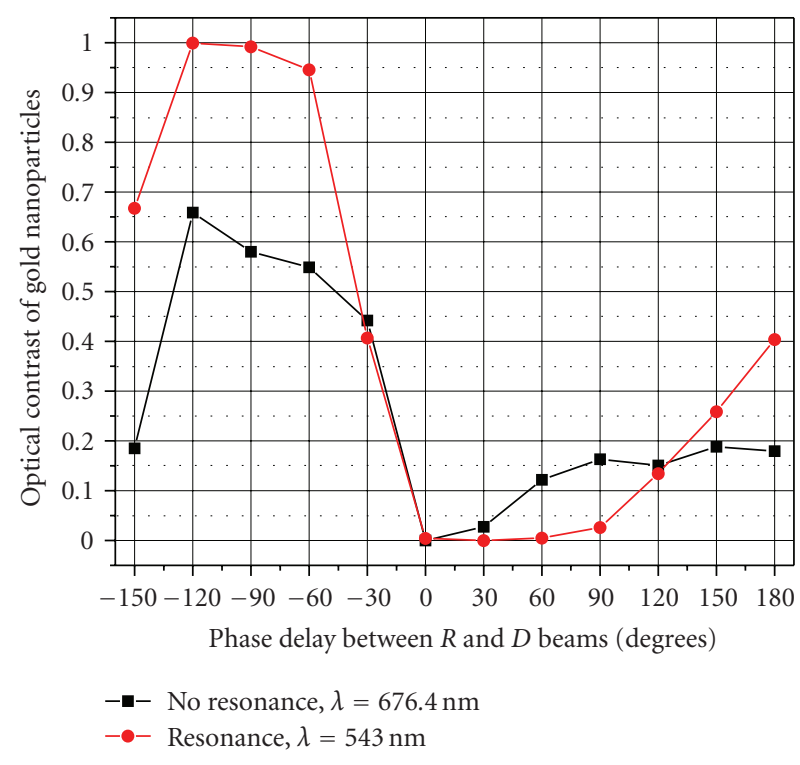

FIgURE 8: Optical contrast due to the gold nanoparticle cluster as a function of the phase offsets between the reference $(R)$ and the diffracted $(D)$ beams of the optical phase microscope.

The correct modeling of the gold NPs required making sure that the FDTD resolution is the same as in the calculation of their optical scattering/absorption properties, that is, $d x=d y=d z=10 \mathrm{~nm}$. This was done by using the built-in features of our FDTD simulation software [14] enabling the possibility for using both nonuniform meshing in the entire computational domain and local hard coding of the resolution of the NPs. The results presented in Figures 68 clearly demonstrate the capability of the proposed FDTD OPCM approach to model the visual effect of gold NPs at nonresonant and resonant conditions. A future extension of this research will be to study the capability of the model to provide valuable insights for the application of gold NPs in optical nanotherapeutics.

\section{CONCLUSIONS}

In this article, we provided simulation results on the application of the FDTD approach to the modeling of the OPCM imaging of gold NPs in singe biological cells. We have first reproduced the effect of optical immersion on the OPCM images of a realistic size cell containing a cytoplasm, a nucleus, and a membrane. The model was then extended to include the presence of a cluster of gold NPs in the cytoplasm at optical immersion conditions as well as the enhancing imaging effect of the optical resonance of the nanoparticles. The results do not allow analyzing the scaling of the NP imaging effect as a function of the number of the NPs in the cluster. However, the validation of the model provides a basis for future studies of OPCM nanobioimaging including the effects of NP cluster size, NP size and number, as well as average dimension between the NPs. To the best of our knowledge, this is the first time that FDTD simulation results were post-processed in a way providing realistic OPCM images of biological cells including a cluster of gold NPs. The results demonstrate the potential of the FDTD approach to model advanced optical nanobioimaging instruments. 


\section{ACKNOWLEDGMENTS}

S. Tanev, J. Pond, and P. Paddon acknowledge the use of the computing resources of WestGrid (Western Canada Research Grid) — a \$50-million project to operate grid-enabled high performance computing and collaboration infrastructure at institutions across Canada. V. V. Tuchin was supported by grants of Federal Agency of Education of RF no. 1.4.06, RNP.2.1.1.4473, RFBR no. 06-02-16740, and Photonics4Life of FP7. The authors are thankful to Dr. Vladimir P. Zharov for the initial discussion on the relevance of the problem of nanoparticle clustering in single cells.

\section{REFERENCES}

[1] P. N. Prasad, Introduction to Biophotonics, chapter 7, John Wiley \& Sons, New York, NY, USA, 2003.

[2] V. V. Tuchin, Tissue Optics: Light Scattering Methods and Instruments for Medical Diagnosis, vol. 166 of Press Monograph Series, SPIE Press, Bellingham, Wash, USA, 2nd edition, 2007.

[3] F. M. Kahnert, "Numerical methods in electromagnetic scattering theory," Journal of Quantitative Spectroscopy and Radiative Transfer, vol. 79-80, pp. 775-824, 2003.

[4] R. Drezek, A. Dunn, and R. Richards-Kortum, "A pulsed finite-difference time-domain (FDTD) method for calculating light scattering from biological cells over broad wavelength ranges," Optics Express, vol. 6, no. 7, pp. 147-157, 2000.

[5] T. Tanifuji and M. Hijikata, "Finite difference time domain (FDTD) analysis of optical pulse responses in biological tissues for spectroscopic diffused optical tomography," IEEE Transactions on Medical Imaging, vol. 21, no. 2, pp. 181-184, 2002.

[6] R. Drezek, M. Guillaud, T. Collier, et al., "Light scattering from cervical cells throughout neoplastic progression: influence of nuclear morphology, DNA content, and chromatin texture," Journal of Biomedical Optics, vol. 8, no. 1, pp. 7-16, 2003.

[7] S. Tanev, V. V. Tuchin, and P. Paddon, "Light scattering effects of gold nanoparticles in cells: FDTD modeling," Laser Physics Letters, vol. 3, no. 12, pp. 594-598, 2006.

[8] S. Tanev, V. V. Tuchin, and P. Paddon, "Cell membrane and gold nanoparticles effects on optical immersion experiments with noncancerous and cancerous cells: finite-difference timedomain modelling," Journal of Biomedical Optics, vol. 11, no. 6, Article ID 064037, 6 pages, 2006.

[9] X. Li, A. Taflove, and V. Backman, "Recent progress in exact and reduced-order modeling of light-scattering properties of complex structures," IEEE Journal on Selected Topics in Quantum Electronics, vol. 11, no. 4, pp. 759-765, 2005.

[10] R. Barer, K. F. A. Ross, and S. Tkaczyk, "Refractometry of living cells," Nature, vol. 171, no. 4356, pp. 720-724, 1953.

[11] B. A. Fikhman, "Microbiological refractometry," Medicine, pp. 81-94, 1967 (Russian).

[12] V. V. Tuchin, Optical Clearing of Tissues and Blood, vol. 154 of Press Monograph Series, SPIE Press, Bellingham, Wash, USA, 2005.

[13] A. Taflove and S. Hagness, Computational Electrodynamics: The Finite-Difference Time Domain Method, Artech House, Boston, Mass, USA, 3rd edition, 2005.

[14] The simulations were performed by the FDTD Solutions ${ }^{\mathrm{TM}}$ software developed by Lumerical Solutions Inc., Vancouver, BC, Canada, http://www.lumerical.com.

[15] K. Sokolov, M. Follen, J. Aaron, et al., "Real-time vital optical imaging of precancer using anti-epidermal growth factor receptor antibodies conjugated to gold nanoparticles," Cancer Research, vol. 63, no. 9, pp. 1999-2004, 2003.

[16] I. H. El-Sayed, X. Huang, and M. A. El-Sayed, "Surface plasmon resonance scattering and absorption of anti-EGFR antibody conjugated gold nanoparticles in cancer diagnostics: applications in oral cancer," Nano Letters, vol. 5, no. 5, pp. 829834, 2005.

[17] N. G. Khlebtsov, A. G. Melnikov, L. A. Dykman, and V. A. Bogatyrev, "Optical properties and biomedical applications of nanostructures based on gold and silver bioconjugates," in Photopolarimetry in Remote Sensing, G. Videen, Ya. S. Yatskiv, and M. I. Mishchenko, Eds., vol. 161 of NATO Science Series II: Mathematics, Physics and Chemistry, pp. 265-308, Kluwer Academic Publishers, Dordrecht, The Netherlands, 2004.

[18] V. P. Zharov, J.-W. Kim, D. T. Curiel, and M. Everts, "Selfassembling nanoclusters in living systems: application for integrated photothermal nanodiagnostics and nanotherapy," Nanomedicine: Nanotechnology, Biology and Medicine, vol. 1, no. 4, pp. 326-345, 2005.

[19] V. P. Zharov, K. E. Mercer, E. N. Galitovskaya, and M. S. Smeltzer, "Photothermal nanotherapeutics and nanodiagnostics for selective killing of bacteria targeted with gold nanoparticles," Biophysical Journal, vol. 90, no. 2, pp. 619-627, 2006.

[20] P. B. Johnson and R. W. Christy, "Optical constants of the noble metals," Physical Review B, vol. 6, no. 12, pp. 4370-4379, 1972. 

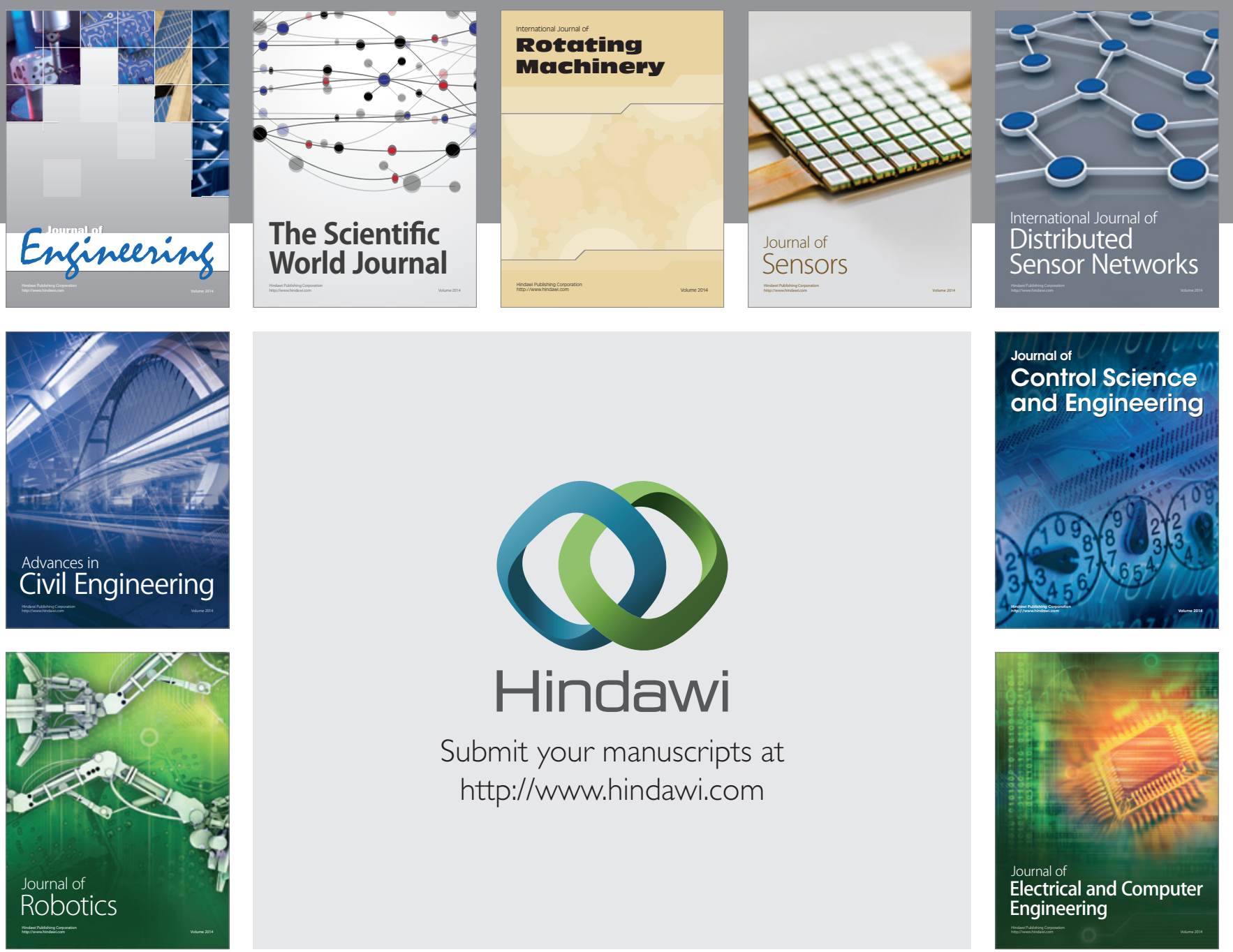

Submit your manuscripts at

http://www.hindawi.com
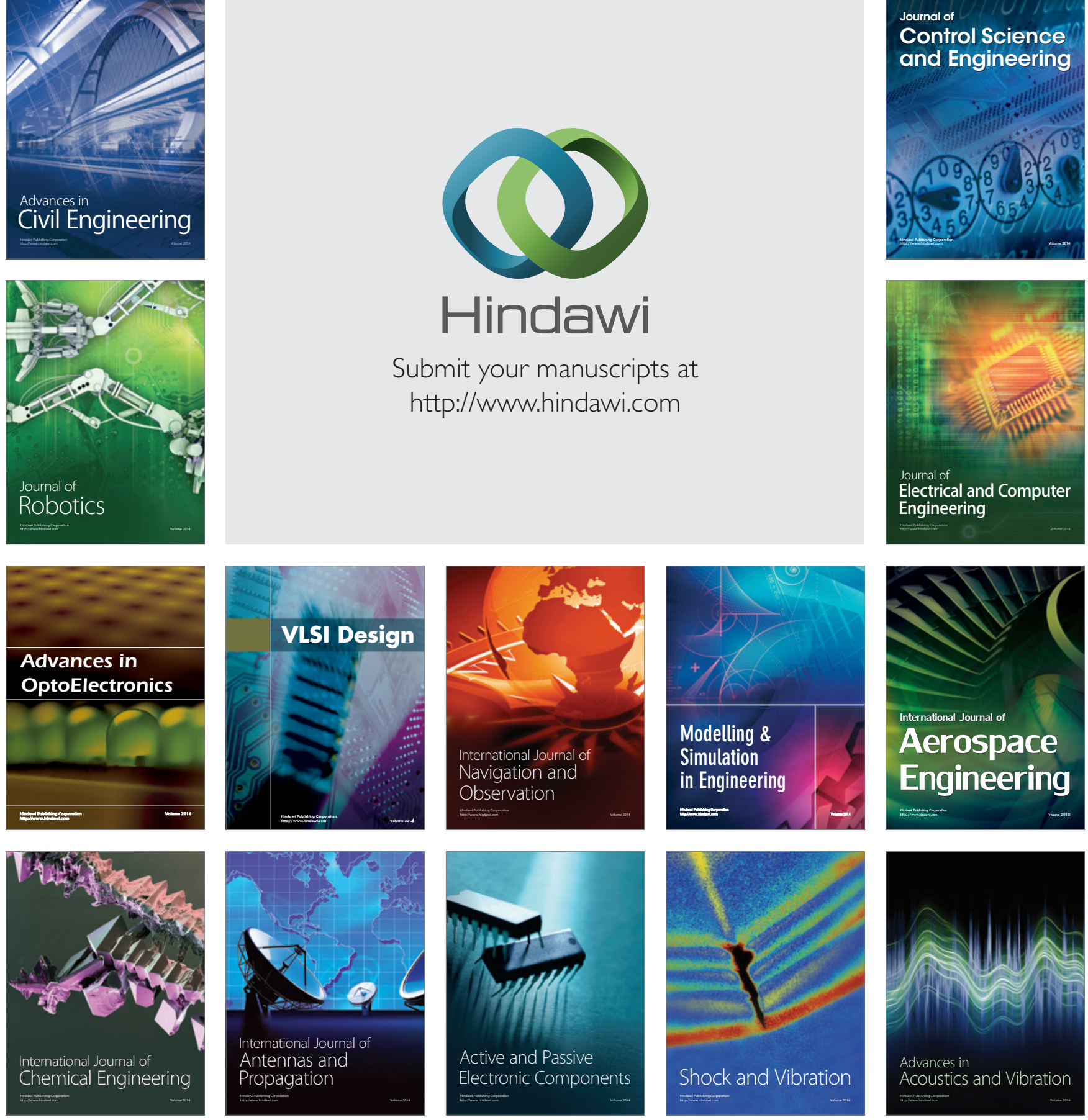